\title{
The Relationship Between Social Support and
} Internet Addiction Among Chinese Adolescents During the COVID-19 Pandemic: A Multiple Mediation Model of Resilience and Post-Traumatic Stress Disorder Symptoms

\author{
Xiumin Cui ${ }^{1,2}$ \\ Xinli $\mathrm{Chi}^{3}$ \\ 'College of Education for the Future, \\ Beijing Normal University, Zhuhai, \\ 51 9087, People's Republic of China; ${ }^{2}$ The \\ Affiliated Kindergarten of Meilian Primary \\ School, Shenzhen, 518035, People's \\ Republic of China; ${ }^{3}$ School of Psychology, \\ Shenzhen University, Shenzhen, 518060, \\ People's Republic of China
}

\begin{abstract}
Introduction: Lockdowns and online homeschooling during the COVID-19 coronavirus pandemic have significantly increased Internet use and adolescents' risk of developing or reinforcing related addictive behaviors. While various theoretical propositions have suggested that social support, resilience, and post-traumatic stress disorder (PTSD) symptoms can predict Internet addiction, no study has yet examined the combined influence of these factors on Internet addiction. This study therefore aimed to investigate the mediating roles of resilience and PTSD symptoms in the relationship between perceived social support and the Internet addiction of Chinese adolescents in the context of COVID-19.

Methods: This study included 2544 adolescents living in the south China city of Qinzhou. The participants completed a self-report questionnaire one week before their return to classes after homeschooling. To examine whether resilience and PTSD symptoms mediated the relationship between social support and Internet addiction, we performed serial mediation analysis using the Model 6 PROCESS macro developed by Hayes (2013).

Results: Social support was negatively and directly associated with Internet addiction and indirectly via resilience, PTSD symptoms, and by the multiple mediation of resilience to PTSD symptoms.

Discussion: The results of this study suggest that, to address Internet addiction, it may be more effective to focus not on addictive behaviors themselves but on individuals' existing mental health. It is important to provide adequate social support to improve the resilience of adolescents, thereby reducing PTSD symptoms and Internet addiction.
\end{abstract}

Keywords: social support, resilience, PTSD symptoms, Internet addiction, COVID-19 pandemic, adolescents

\section{Introduction}

With the continuous development of the Internet in recent decades, Internet use has become an indispensable part of adolescents' lives, but, simultaneously, Internet addiction has become an increasing concern for both researchers and practitioners. This behavioral manifestation involves excessive dependence on the Internet and its applications, which has a detrimental influence on an individual's psychological and social functioning. ${ }^{1,2}$ Research has shown that Internet addicts are more likely to
Correspondence: Xinli Chi

School of Psychology, Shenzhen

University, 1688 Nanhai Avenue, Nanshan

District, Shenzhen, Guangdong Province,

People's Republic of China

Email xinlichi@I26.com 
have mental health problems such as depression, stress, and anxiety, potentially leading to intrapersonal difficulties, academic failure, and family disruption. ${ }^{3,4}$ In 2020, of 904 million Internet users in China, 175 million were under 19 years old, ${ }^{5}$ and a 2019 study showed that the prevalence of Internet addiction among Chinese adolescents had reached $15.3 \%{ }^{6}$

Since January 2020, the COVID-19 pandemic and consequent lockdowns have disrupted people's normal activities. To effectively protect students' health and safety, China's Ministry of Education postponed the opening of schools and implemented a "classes are suspended but learning continues" approach by offering online courses. $^{7}$ These initiatives have, combined with limited face-to-face interaction and increased Internet-based homeschooling, significantly expanded Internet use and posed potential risks for adolescents of developing or reinforcing Internet-related disorders, such as persistent online gaming ${ }^{8}$ and Internet addiction. ${ }^{9}$ Accordingly, there is an urgent need for studies to better understand the influencing factors and underlying mechanisms of Internet addiction in order to provide recommendations for its prevention and treatment during the COVID-19 pandemic.

\section{Social Support and Internet Addiction}

Social support can potentially protect against Internet addiction. ${ }^{10}$ This paper defines social support as an individual's perceived physical and/or psychological resources that are available in the surrounding environment from, for example, family, friends, or other significant people. ${ }^{11}$ According to Davis's cognitive-behavioral model, a lack of perceived social support can lead to excessive dependence on the Internet in a quest for equivalent assistance in the virtual world, which may be accompanied by problematic Internet use. ${ }^{1}$ Moreover, according to the psychological decompensation model, ${ }^{12,13}$ Internet overuse can compensate adolescents for difficulties in their offline psychological development; that is, when psychological development is hindered by a lack of adequate social support, Internet activities can provide pathological compensation, potentially resulting in addictive behaviors. ${ }^{10}$ Empirical studies have shown that, because Internet addicts receive less social support than non-addicts, they look to their virtual networks for additional support. ${ }^{14,15}$ Insufficient social support can cause individuals to satisfy their psychological needs from inappropriate sources, which can lead to the development of Internet addiction. ${ }^{12,13}$ By contrast, adequate social support can reduce the incidence of problematic Internet use. ${ }^{10,16}$ According to the above-mentioned cognitive-behavioral and psychological decompensation models, we hypothesized that social support would be negatively correlated with Internet addiction among Chinese adolescents during the COVID-19 crisis (Hypothesis 1).

\section{The Mediating Role of Resilience}

The predictive mechanisms of social support for Internet addiction remain unclear; however, behavior synthesis theory asserts that the impact of an individual's social environment on problem behaviors is mediated by the individual's personal qualities, ${ }^{17}$ and resilience may be an important positive mediator between social support and problem behavior. ${ }^{18-20}$ This paper defines resilience as an individual's capacity to recover from adversity, trauma, threats, or significant sources of stress. ${ }^{21}$ Theoretically, the development of resilience depends on a combination of the required resources being provided by the individual's community and the effective utilization of those resources by the individual. ${ }^{18,22}$ Previous research has demonstrated that high levels of social support from family and friends can enhance feelings of belonging and solidarity, foster active problem-solving, and thereby increase resilience. ${ }^{19}$ Consequently, a high degree of resilience may help an individual to deal with stress and resist Internet addiction. ${ }^{23}$ Studies have indicated that resilience predicts adolescents' preferences for online content ${ }^{24}$ and is negatively associated with problematic Internet use $e^{4,25}$ Based on this previous work, Hypothesis 2 asserted that resilience has a mediating effect on the relationship between social support and Internet addiction.

\section{The Mediating Role of Post-Traumatic Stress Disorder (PTSD)}

For adolescents who experience profoundly difficult life events, such as the COVID-19 crisis, PTSD may be a key factor in the development of Internet addiction. PTSD is a serious and chronic response to traumatic experiences, with typical symptoms including memory intrusion, reminder avoidance, negative thoughts or moods, and hypervigilance. $^{21}$ The global public health emergency caused by the COVID-19 pandemic disrupted adolescents' normal activities, and their prolonged exposure to stress during the crisis gave rise to mental health challenges, including PTSD symptoms. ${ }^{26}$ 
Individuals who suffer from PTSD are known to be vulnerable to behavioral addictions, including substance abuse, eating disorders, and problematic smartphone use. $^{27-29}$ The self-medication theory ${ }^{30}$ has suggested that people with PTSD develop maladaptive coping strategies, including addictive behaviors that can function to reduce intrusive symptoms and facilitate escape from or avoidance of perceived stressors. ${ }^{29,31}$ According to this approach, Internet dependence serves an avoidance function for adolescents with PTSD, who may immerse themselves in the virtual world to avoid the negative emotions and psychological pain of symptoms. ${ }^{3,32}$ Several empirical studies have directly linked PTSD symptoms to Internet addiction; ${ }^{33,34}$ for example, a study of students exposed to the Sewol ferry disaster in South Korea found that their Internet addiction was significantly associated with PTSD symptoms. ${ }^{33}$

It is vital to identify the factors that may prevent the occurrence of PTSD symptoms, and social support is considered one of the strongest predictors in this regard. ${ }^{35}$ The social support deterioration deterrence model $^{36}$ claims that this kind of support can mitigate adverse psychological symptoms following a traumatic event, and its importance for alleviating PTSD symptoms among children and adolescents became evident in the contexts of Hurricane Katrina ${ }^{37}$ and the Sri Lankan tsunamis. ${ }^{38}$ Compared with people who receive low social support, traumatized individuals with strong support networks report fewer negative psychological outcomes and less severe PTSD symptoms. ${ }^{39}$ Additionally, social support has proved to play a key role in the treatment of PTSD $;{ }^{40}$ for example, Gros et $\mathrm{al}^{29}$ found that social support, by providing regular positive experiences, helped to alleviate distress and habituate individuals to their memories of traumatic events. Since social support may reduce the severity of PTSD symptoms and the need for avoidance strategies, decreasing an individual's vulnerability to addictive behaviors, we proposed that social support can reduce Internet addiction specifically by relieving PTSD symptoms. These rationales together led to the formulation of Hypothesis 3: PTSD has a mediating effect on the relationship between social support and Internet addiction.

\section{The Relationship Between Resilience and PTSD}

According to the psychological resources theory developed by Hobfoll, some individuals deal with adversity better than others due to their rich psychological resources, ${ }^{41}$ which can be influenced by various interacting factors, such as personality traits, ${ }^{42}$ parenting style, ${ }^{43}$ and access to social support. ${ }^{44}$ In particular, social support can enhance individuals' optimism, self-confidence, and positive attitudes toward difficult circumstances, which can themselves be seen as important psychological resources. Resilience, as a core component of psychological resources, may protect against psychological distress, including PTSD symptoms, ${ }^{45,46}$ and specifically help individuals to adapt effectively to stressful events through various beliefs, attitudes, and coping strategies. ${ }^{47}$ Individuals with high resilience are less prone to developing PTSD symptoms, ${ }^{31}$ reducing their risk of later developing of Internet addiction. ${ }^{48}$ We therefore proposed that social support can lead to higher levels of resilience and, in turn, reduce PTSD symptoms, thus decreasing vulnerability to Internet addiction (Hypothesis 4).

\section{The Present Study}

Although various theories could explain how social support affects Internet addiction, the underlying mechanism of this relationship has not yet been empirically examined. To the best of our knowledge, no study has addressed the combined role of social support, resilience, and PTSD symptoms in the development of Internet addiction. Furthermore, it is crucial to extend such research to adolescents because of their increased vulnerability to Internet addiction in a critical developmental period of their lives. ${ }^{6}$ Consequently, the primary aim of the present study was to explore the potential mediating role of resilience and PTSD symptoms in the relationship between social support and Internet addiction among adolescents during the COVID-19 pandemic. The results highlighted the functional roles of social support, resilience, and intervention strategies for adolescents with PTSD symptoms and Internet addiction.

\section{Methods}

\section{Participants and Procedures}

This study involved adolescents living in the south China city of Qinzhou who completed a self-report questionnaire between late April and early May 2020 - one week before they were due to return to physical classes after the coronavirus outbreak. The local education authority gave its official approval to conduct the study in a public school. Participants and their guardians were informed about the study's purpose 
and the voluntary nature of participation before the survey was administered, and all participants and their parents gave their signed informed consent to the researchers. The survey was administered online to ensure COVID-19 safety. A total of 2873 students participated in the survey, 2544 (88.55\%) of whom provided valid responses. The sample consisted of $1086(42.7 \%)$ and $1458(57.3 \%)$ male and female respondents, respectively. There were 1428 (56.13\%) Grade 10 and 1116 (43.87\%) Grade 11 students with a mean age of 16.49 years $(\mathrm{SD}=0.75$, range 13-20). The Human Research Ethics Committee of Shenzhen University approved the recruitment and data collection procedures under reference 2020005.

\section{Measures}

\section{Social Support}

The Social Support Rating Scale (SSRS) was used to measure social support. ${ }^{49}$ This is a 10 -item instrument that assesses support across three categories: subjective, objective, and available. All items were rated from 1 to 4 , with higher scores indicating greater social support. It has been used widely in the Chinese population and has shown high reliability ( $\alpha=0.69$ ), structure validity ( $r s$ between 0.462 and 0.664 ) and content validity ( $r s$ between 0.724 and 0.836$){ }^{53}$ The modified inventory for the present sample had good internal reliability $(\alpha=0.86)$ and an acceptable confirmatory fit index $(\mathrm{CFI}=0.951$, TLI $=0.91$, RMSEA $=0.06$, SRMR $=0.04)$.

\section{Resilience}

Resilience was assessed using the abridged ConnorDavidson Resilience Scale (CD-RISC), which primarily assesses an individual's capacity for problem-solving, positive thinking, emotional control, and toughness when confronted by pressure, change, illness, or failure. ${ }^{50}$ It comprises 10 items, each answered on a 5-point scale from 0 (not true at all) to 4 (true nearly all the time), with higher values indicating stronger resilience. The reliability coefficient of the CD-RISC was 0.85 . Its validity was demonstrated by the fact the scale had expected correlation with childhood maltreatment and psychiatric symptoms $(r>0.72)$. In this study, the internal reliability was good ( $\alpha=0.94)$, as was the confirmatory fit index $(\mathrm{CFI}=0.98, \mathrm{TLI}=0.96, \mathrm{RMSEA}=0.08, \mathrm{SRMR}=0.03)$.

\section{PTSD Symptoms}

We used the abbreviated PTSD Checklist-Civilian Version $(\mathrm{PCL}-\mathrm{C})^{51}$ to measure the severity of PTSD symptoms experienced by the participants over the previous month. The reliability $(\alpha=0.94)$ and validity $(r>$ $0.75)$ of the PCL-C have been established. This instrument consists of three subscales: reexperiencing symptoms (two items), avoidance symptoms (two items), and hyperarousal symptoms (two items). All items were rated on a 5-point scale ranging from 1 (not at all) to 5 (extremely), and the final score was the sum of the responses. An individual was considered to have PTSD symptoms if their total score was 14 or higher. For this research, we adjusted the questions to specifically assess the influence of the COVID-19 crisis; the scale had good internal reliability $(\alpha=0.82)$ and a good confirmatory fit index (CFI $=0.99$, $\mathrm{TLI}=0.99, \mathrm{RMSEA}=0.01, \mathrm{SRMR}=0.004)$.

\section{Internet Addiction}

The 10-item Chinese version ${ }^{52}$ of the self-rated Diagnostic Questionnaire for Internet Addiction ${ }^{2}$ was used to assess the presence of Internet addiction. The Cronbach's $\alpha$ of the original scale was 0.75 and it also exhibited meaningful correlations with theoretically-relevant constructs such as "self-assessed as addicted to the Internet" $(r=0.423)$ and "self-assessed as excessively use or depend on the Internet" ( $r=0.412)$ in the original study. Participants responded "Yes" or "No" to statements according to whether or not they had experienced the situations described, and those who responded positively to four or more of the listed behaviors were classified as Internet addicts. Its internal reliability was good $(\alpha=0.82)$ in this study. Furthermore, the confirmatory fit index (CFI $=0.97$, $\mathrm{TLI}=0.96, \mathrm{RMSEA}=0.05, \mathrm{SRMR}=0.03$ ) indicated the good validity of the scale for the present sample.

\section{Statistical Analyses}

The data analysis was conducted using SPSS version 22.0 (IBM Corp., Armonk, NY, United States), and the relationships between social support, resilience, PTSD symptoms (total score and three dimensions), and Internet addiction were examined using Pearson's correlation analysis. To determine whether resilience and PTSD symptoms mediated the relationship between social support and Internet addiction, we performed serial mediation analysis using the Model 6 PROCESS macro developed by Hayes. ${ }^{54}$ The $\beta$ coefficients reported in this study are standardized coefficients and can thus be compared to determine the relative strength of the different variables within the model. In particular, the bootstrapping method was used to evaluate significant effects. 
Table I Descriptive Statistics and Correlation Analyses for Social Support, Resilience, PTSD, and Internet Addiction

\begin{tabular}{|c|c|c|c|c|c|c|c|c|c|}
\hline Variables & $\mathbf{M}$ & SD & $\mathbf{I}$ & 2 & 3 & 4 & 5 & 6 & 7 \\
\hline I. Social support & 42.11 & 7.39 & 1 & & & & & & \\
\hline 2. Resilience & 35.85 & 8.06 & $0.387^{* * * *}$ & I & & & & & \\
\hline 3. PTSD & 10.97 & 4.04 & $-0.257^{* * * *}$ & $-0.363 * * *$ & 1 & & & & \\
\hline 4. Reexperiencing symptoms & 3.31 & 1.49 & $-0.113^{* * *}$ & $-0.226 * * *$ & $0.792 * * *$ & 1 & & & \\
\hline 5. Avoidance symptoms & 3.43 & 1.55 & $-0.200 * * *$ & $-0.249 * * *$ & $0.858 * * *$ & $0.635 * * *$ & I & & \\
\hline 6. Hyperarousal symptoms & 4.23 & 1.90 & $-0.296 * * *$ & $-0.392^{* * *}$ & $0.808^{* * * *}$ & $0.386 * * *$ & $0.513^{* * * *}$ & 1 & \\
\hline 7. Internet addiction & 3.51 & 2.78 & $-0.247^{* * *}$ & $-0.267 * * *$ & $0.342 * * *$ & $0.183^{* * *}$ & $0.204^{* * *}$ & $0.420^{* * * *}$ & I \\
\hline
\end{tabular}

Note: $* * * p<0.001$.

\section{Results}

\section{Preliminary Analysis}

The descriptive statistics (means and SDs) and results of the correlation analysis are shown in Table 1. In this study, the prevalence of participants diagnosed with Internet addiction and PTSD symptoms was $45.32 \%$ (1153 participants) and $22.96 \%$ (584 participants), respectively. Social support was positively correlated with resilience $(r=$ 0.387, $p<0.001)$, but negatively correlated with PTSD symptoms $(r=-0.257, p<0.001)$ and Internet addiction $(r=-0.247, p<0.001)$. Resilience was negatively correlated with both PTSD symptoms $(r=-0.363, p<0.001)$ and Internet addiction $(r=-0.267, p<0.001)$, whereas
PTSD symptoms were positively correlated with Internet addiction $(r=0.342, p<0.001)$. Furthermore, we found that all three PTSD symptom clusters were positively correlated with Internet addiction. Specifically, hyperarousal symptoms were strongly correlated with Internet addiction $(r=0.420, p<0.001)$, followed by avoidance symptoms $(r=0.204, p<0.001)$ and reexperiencing symptoms $(r=0.183, p<0.001)$.

\section{Testing for Multiple Mediation}

We applied the Model 6 PROCESS template for SPSS and SAS (http://www.afhayes.com/) to evaluate multiple mediation. As shown in Table 2, after controlling for gender

Table 2 Regression Results of the Mediation Analysis

\begin{tabular}{|c|c|c|c|c|c|c|c|}
\hline Outcome Variables & Independent Variables & $\mathbf{R}$ & $\mathbf{R}^{2}$ & $\mathbf{F}$ & $d f$ & $\beta$ & $\boldsymbol{t}$ \\
\hline \multirow[t]{2}{*}{ Internet addiction } & & 0.248 & 0.062 & $55.530 * * *$ & 3 & & \\
\hline & $\begin{array}{l}\text { Gender } \\
\text { Age } \\
\text { Social support }\end{array}$ & & & & & $\begin{array}{c}0.049 \\
0.006 \\
-0.247\end{array}$ & $\begin{array}{c}1.247 \\
0.228 \\
-12.843 * * *\end{array}$ \\
\hline Resilience & $\begin{array}{l}\text { Gender } \\
\text { Age } \\
\text { Social support }\end{array}$ & 0.419 & 0.176 & $180.358 * * *$ & 3 & $\begin{array}{c}-0.322 \\
0.007 \\
0.387\end{array}$ & $\begin{array}{c}-8.85 I^{* * *} \\
0.283 \\
21.467^{* * *}\end{array}$ \\
\hline PTSD & $\begin{array}{l}\text { Gender } \\
\text { Age } \\
\text { Social support } \\
\text { Resilience }\end{array}$ & 0.395 & 0.156 & $117.329 * * *$ & 4 & $\begin{array}{l}0.160 \\
0.065 \\
-0.143 \\
-0.296\end{array}$ & $\begin{array}{c}4.284 * * * \\
2.684 \\
-7.225 * * * \\
-14.719 * * *\end{array}$ \\
\hline Internet addiction & $\begin{array}{l}\text { Gender } \\
\text { Age } \\
\text { Social support } \\
\text { Resilience } \\
\text { PTSD }\end{array}$ & 0.395 & 0.156 & $93.977 * * *$ & 5 & $\begin{array}{l}-0.060 \\
-0.010 \\
-0.130 \\
-0.124 \\
0.268\end{array}$ & $\begin{array}{c}-1.598 \\
-0.422 \\
-6.488^{* * *} \\
-5.928 * * * \\
3.503^{* * *}\end{array}$ \\
\hline
\end{tabular}

Notes: $\mathrm{N}=2544$; bootstrap sample $=5000 ; \beta$ values are standardized coefficients and can be compared to determine the relative strength of the different variables within the model. Gender was dummy coded as $0=$ male and I = female; $* * * p<0.00 \mathrm{I}$. 
Table 3 Bootstrapping Indirect Effects and 95\% Cls for the Multiple Mediation Analysis

\begin{tabular}{|l|c|c|c|c|c|}
\hline \multirow{2}{*}{ Indirect Effect } & Estimated Effect & \multirow{2}{*}{ Boot SE } & \multicolumn{2}{|c|}{ 95\% Cl } & \multirow{2}{*}{ Relative Mediation Effect } \\
\cline { 3 - 5 } & & & Boot LLCl & Boot ULCl & \\
\hline Total & -0.117 & 0.011 & -0.139 & -0.096 & $47.39 \%$ \\
IndI & -0.048 & 0.010 & -0.067 & -0.030 & $19.44 \%$ \\
Ind2 & -0.038 & 0.007 & -0.051 & -0.026 & $15.56 \%$ \\
Ind3 & -0.031 & 0.004 & -0.038 & -0.024 & $12.39 \%$ \\
CI & -0.010 & 0.012 & -0.034 & 0.014 & \\
C2 & -0.017 & 0.010 & -0.038 & 0.003 & \\
C3 & -0.008 & 0.007 & -0.022 & 0.007 & \\
\hline
\end{tabular}

Notes: $\mathrm{N}=2544$; bootstrap sample $=5000$; Ind I = social support $\rightarrow$ resilience $\rightarrow$ Internet addiction; Ind2 $=$ social support $\rightarrow$ PTSD $\rightarrow$ Internet addiction; Ind3 $=$ social support $\rightarrow$ resilience $\rightarrow$ PTSD $\rightarrow$ Internet addiction; $\mathrm{Cl}=$ IndI-Ind2; C2 $=$ Ind I-Ind3; C3 = Ind2-Ind3.

Abbreviations: Boot $\mathrm{LLCl}$, boot lower limit confidence interval; Boot $\mathrm{ULCl}$, boot upper limit confidence interval.

and age, social support was a negative predictor of Internet addiction in the absence of a mediator $(\beta=-0.247, p<$ $0.001)$; it was positively associated with resilience $(\beta=$ $0.387, p<0.001)$ and negatively associated with PTSD symptoms $(\beta=-0.143, p<0.001)$. However, resilience negatively predicted both PTSD symptoms $(\beta=-0.296$, $p<0.001)$ and Internet addiction $(\beta=-0.124, p<0.001)$, and PTSD symptoms were positively associated with Internet addiction $(\beta=0.268, p<0.001)$.

The results of bias-corrected percentile bootstrap analysis revealed significant indirect effects of resilience and PTSD on the relationship between social support and Internet addiction (Table 3 and Figure 1). The total standardized indirect effect was $-0.117(95 \%$; CI $[-0.139$, $-0.096])$. The indirect effects followed three paths: social support $\rightarrow$ resilience $\rightarrow$ Internet addiction (Ind1, estimated effect $=-0.048$ ); social support $\rightarrow$ PTSD $\rightarrow$ Internet addiction (Ind2, estimated effect $=-0.038$ ); and social support $\rightarrow$ resilience $\rightarrow$ PTSD $\rightarrow$ Internet addiction
(Ind3, estimated effect $=-0.031$ ). The mediation effects of the three paths accounted for $19.44 \%, 15.56 \%$, and $12.39 \%$ of the total effect on the relationship between social support and Internet addiction, respectively. Additionally, none of the empirical 95\% CI values overlapped with zero, meaning that the mediation effects were significant. Subsequent pairwise comparison of the indirect effects showed no significant differences between the three paths, since all the $95 \%$ CI values overlapped with zero.

\section{Discussion}

This study examined the relationship between social support and Internet addiction, as well as the mediating roles of resilience and PTSD symptoms, among Chinese adolescents during the recent COVID-19 crisis. After controlling for gender and age, our findings showed that social support was negatively and directly associated with Internet addiction and indirectly via resilience, PTSD

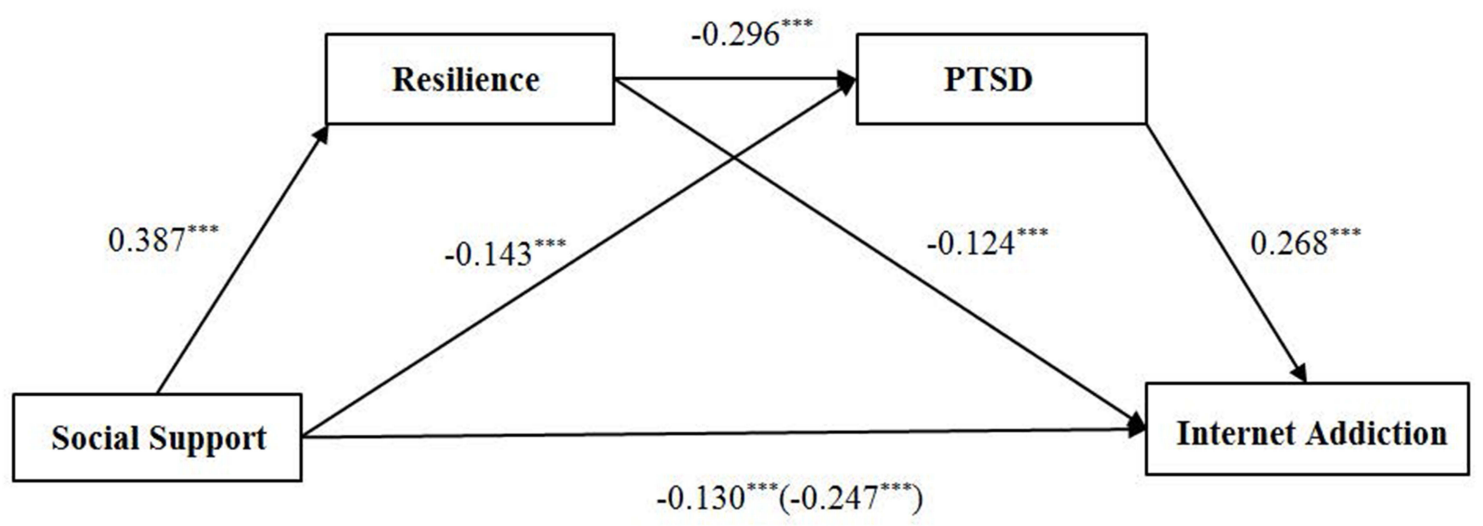

Figure I A multiple mediation model of the association between social support and Internet addiction through resilience and PTSD symptoms. Standardized regression coefficients are shown on the paths $\left(*^{* *} p<0.00 \mathrm{I}\right)$. The coefficient of the direct path from social support to Internet addiction in the absence of a mediator is presented in parentheses. 
symptoms, and by the multiple mediation of resilience to PTSD symptoms.

More specifically, consistent with previous research, the direct effect observed in this study indicated that the more social support an individual receives, the lower their Internet addiction is likely to be. ${ }^{10,16}$ This finding supports the view that adequate social support is a positive resource for lessening adolescents' problematic behavior. ${ }^{55}$ The high COVID-19 transmission and mortality rates ${ }^{56}$ presented particular challenges for adolescents because of adolescents' relative psychological immaturity. Objective and subjective social support may provide young people with the resources they need to maintain emotional wellbeing by developing coping strategies for COVID-19related issues, instead of avoiding difficulty and stress by resorting to online networks. Ultimately, strong social support may thus reduce excessive dependence on the Internet. $^{10,57}$ By contrast, according to the psychological decompensation model of problematic internet use, ${ }^{12,13}$ adolescents without adequate social support may feel emotionally rejected and consequently seek self-affirmation from other sources, which can push them to engage in excessive Internet use and develop Internet addiction. $^{12,16,58,59}$

This study showed that resilience is a significant mediator of the relationship between social support and Internet addiction, consistent with previous studies. ${ }^{16,60}$ In response to the COVID-19 crisis, individuals with high levels of social support tended to experience increased feelings of belonging and solidarity, which helped them to face the challenges of the pandemic and thereby develop resilience. ${ }^{61}$ Specifically, as a major source of social support, consistent support from parents can help adolescents to maintain emotional and spiritual well-being. In particular, if parents set a good example in dealing with the adverse circumstances caused by the pandemic, adolescents may cope effectively with stress and develop high levels of resilience. Additionally, encouragement and care from peers and teachers can enable adolescents to control the negative feelings associated with stressful circumstances, helping them to feel less alone in facing problems and consequently improving their psychological resilience. Resilient adolescents are more capable of dealing with COVID-19-related fears and experiencing positive emotions and thoughts, all of which allow them to adapt to lockdown conditions, readjust to online homeschooling, manage their use of electronic devices, and reduce Internet addiction. ${ }^{25,62}$
This study also found that the presence of PTSD symptoms related to COVID-19 was another significant mediator of the association between social support and Internet addiction, extending previous findings about this relationship. $^{29,32}$ Consistent with the social support deterioration deterrence model, ${ }^{36}$ adolescents who receive support from parents and friends are more likely to identify their feelings correctly, respond to negative emotions adaptively, and cope with stress actively, thereby relieving psychological difficulties such as those experienced in PTSD. ${ }^{29}$ By contrast, adolescents who do not have adequate social support can feel lonelier and more helpless when facing the lifestyle changes and stressful circumstances caused by the pandemic, in turn making them more vulnerable to developing PTSD symptoms. ${ }^{63}$ Furthermore, previous research has shown that individuals who report PTSD symptoms are likely to deal with their negative experiences by seeking safe, calm environments as a means of "self-medication." 3 Consistent with this, our study found a positive correlation between reexperiencing, avoidance, and hyperarousal PTSD symptom clusters and Internet addiction. During the COVID-19 pandemic, adolescents with reexperiencing and avoidance symptoms experienced painful emotional reactions when thinking about COVID-19-related scenarios, and limited face-toface interaction and Internet-based education increased Internet adolescents' Internet use, making virtual networks a convenient place for getting attention and relieving psychological distress. The same was true for adolescents with hyperarousal symptoms, who exhibited hypervigilance, had difficulty concentrating, and tended to use the Internet to alleviate these symptoms. The intense and emotionally distressing experiences associated with PTSD can impair individuals' ability to control their noneducational use of the Internet, because the immediately rewarding experiences facilitated by the Internet can reduce or distract them from psychological struggles, thus increasing their risk of Internet addiction. ${ }^{28}$

The relationship between social support and Internet addiction was partially mediated by resilience to PTSD symptoms. According to the biopsychosocial model, ${ }^{64}$ Internet addiction is a particularly difficult problem influenced by social, psychological, and physiological factors. Environments that are loving, emotionally responsive, consistent, and reliable may offer young people the opportunity to master the challenges and stresses resulting from unprecedented world events, such as the COVID-19 pandemic, and the corresponding hardships, thereby 
developing their resilience. ${ }^{19}$ Such resilient adolescents are more likely to view the unfavorable circumstances of the pandemic as temporary, limited in scope, and surmountable $^{4}$ and thus build more successful defenses against negative feelings about the pandemic, experience positive emotions, and reduce their PTSD symptoms, ultimately decreasing their risk of seeking refuge in the virtual world and developing Internet addiction.

This work has several limitations. First, the study relied on a self-report survey that only reflected the adolescents' perspectives. It would be worthwhile to include information from multiple informants, including parents, peers, and teachers, to reduce potential bias in estimating the factor associations. Second, due to the background and aim of our study, the sample included only Chinese adolescents influenced by the COVID-19 pandemic. Accordingly, generalization of the results to adolescents with PTSD symptoms arising from other stressful or traumatic events should be cautious. Third, although three PTSD symptom clusters relate to Internet addiction, we only examined the mediating effect of PTSD symptoms overall. Further research should investigate the different mediating effects of each PTSD symptom to determine which symptoms correlate most strongly with Internet addiction. Finally, the cross-sectional data only provided a snapshot of the relationship between the studied factors and Internet addiction. Further longitudinal studies would contribute to understanding the casual associations between the behaviors and their underlying factors.

\section{Conclusion}

Despite these limitations, this study is the first to explore the interplay between social support, resilience, PTSD symptoms, and Internet addiction. The results therefore provide further evidence of the direct effects of social support on Internet addiction and indicate that such behavior could be alleviated by social support through the two chain mediators of resilience and PTSD symptoms. It would be helpful to explore the causal associations between the variables in a further longitudinal study. In terms of interventions to reduce problematic Internet behavior among adolescents during unprecedented world events such as the recent pandemic, this study highlights that it may be more effective to focus not on the addictive behavior itself but on its underlying mechanisms, including social support, resilience, and PTSD symptoms. Specifically, educators and parents can make efforts to foster supportive environments that provide adequate resources and emotional support for adolescents. Additionally, helping young people to enhance their resilience and encouraging them to use active coping skills in response to traumatic events may reduce the occurrence of PTSD symptoms and, consequently, Internet addiction.

\section{Ethical Approval}

All procedures were approved by the Research Ethics Committee of Shenzhen University and this study complied with the Declaration of Helsinki.

\section{Informed Consent}

Informed consent was obtained from all individual participants and their guardians in this study.

\section{Author Contributions}

Both authors made a significant contribution to the work reported, whether that is in the conception, study design, execution, acquisition of data, analysis and interpretation, or in all these areas; took part in drafting, revising or critically reviewing the article; gave final approval of the version to be published; have agreed on the journal to which the article has been submitted; and agree to be accountable for all aspects of the work.

\section{Funding}

This research was funded by Guangdong Basic and Applied Basic Research Foundation, grant numbers 2021A1515011330; Shenzhen Education Science Foundation, grant number zdfz17006; Shenzhen Philosophy and Social science Foundation, grant number SZ2018C001.

\section{Disclosure}

Authors declare that they have no conflict of interest.

\section{References}

1. Davis RA. A cognitive-behavioral model of pathological Internet use. Comput Human Behav. 2001;17(2):187-195. doi:10.1016/S07475632(00)00041-8

2. Young KS. Internet addiction: the emergence of a new clinical disorder. Cyberpsychol Behav. 1999;1(3):237-244. doi:10.1089/ cpb.1998.1.237

3. Evren B, Dalbudak E, Evren C, Ozen S. The relationship of internet addiction symptom severity with post-traumatic stress disorder symptoms and impulsivity among Turkish university students. Psychiat Clin Psych. 2018;29(1):83-89.

4. Nam CR, Lee DH, Lee JY, et al. The role of resilience in internet addiction among adolescents between sexes: a moderated mediation model. J Clin Med. 2018;7(8):222-235. doi:10.3390/jcm7080222 
5. The 45th China statistical report on Internet development [homepage on the Internet]. Beijing: China Internet Network Information Center; 2020. Available from: http://cnnic.net.cn/hlwfzyj/hlwxzbg/hlwtjbg/ 202004/t20200428 70974.htm. Accessed May 26, 2020.

6. Chi X, Hong X, Chen X. Profiles and sociodemographic correlates of Internet addiction in early adolescents in southern China. Addict Behav. 2020;10(6):385-399.

7. Notice of the Ministry of Education on the extension of the spring semester in 2020 [homepage on the Internet]. Beijing: Ministry of Education of the People's Republic of China; 2020. Available from: http://www.moe.gov.cn/jyb_xwfb/gzdt_gzdt/s5987/202001/ t20200127_416672.html. Accessed May 26, 2020.

8. King DL, Delfabbro PH, Billieux J, Potenza MN. Problematic online gaming and the COVID-19 pandemic. J Behav Addict. 2020;9 (2):184-186. doi:10.1556/2006.2020.00016

9. Sun Y, Li Y, Bao Y, et al. Brief report: increased addictive internet and substance use behavior during the COVID-19 pandemic in China. Am J Addict. 2020;29(4):268-270. doi:10.1111/ajad.13066

10. Wang S, Zhang D. The impact of perceived social support on students' pathological internet use: the mediating effect of perceived personal discrimination and moderating effect of emotional intelligence. Comput Human Behav. 2020;106:247-286. doi:10.1016/j. chb. 2020.106247

11. Vaux A. Social Support: Theory, Research, and Intervention. SanFrancisco: Praeger publishers; 1998.

12. Gao W, Chen Z. A study on psychopathology and psychotherapy of Internet addiction. Adv Psychol Sci. 2006;14:596-603.

13. Gao W, Zhu Z, Chen Z, Liu Z, Gao J The psychological mechanism of Internet addiction: "Psychological decompensation hypothesis". Paper presented at: 9th Professional Committee Meeting for Adolescents' Mental Health in Chinese Association for Mental Health; October 26; 2005; Hohhot, Inner Mongolia, China.

14. Gao F, Xu J, Ren Y, Han L. The relationship between internet addiction and aggression: multiple mediating effects of life events and social support. J Psychol Res. 2016;6(1):42-49.

15. Karaer Y, Akdemir D. Parenting styles, perceived social support and emotion regulation in adolescents with internet addiction. Compr Psychiatry. 2019;92:22-27. doi:10.1016/j.comppsych.2019.03.003

16. Tas I. Association between depression, anxiety, stress, social support, resilience and Internet addiction: a structural equation modelling. Malays Online J Educ Technol. 2019;7(3):1-10. doi:10.17220/ mojet.2019.03.001

17. Zhang D, Zhu Z, Liu G, Li Y. The relationship between social support and problem behaviors of adolescents: the chain mediating role of psychological sushi and self-esteem. J Southwest Univ. 2019;45(1):99-104.

18. Salami SO. Moderating effects of resilience, self-esteem and social support on adolescents' reactions to violence. Asian Soc Sci. 2010;6 (12):101-135. doi:10.5539/ass.v6n12p101

19. Sippel LM, Pietrzak DS, Charney LC, Mayes SM. How does social support enhance resilience in the trauma-exposed individual? Ecol Soc. 2015;20(4):10-36. doi:10.5751/ES-07832-200410

20. Xin Y, Bai K, Chen X, Zhu D, Liu C. The effect of social support on post-traumatic growth of adolescents: the mediating role of resilience. Stud Psychol Behav. 2019;17(6):817-823.

21. American Psychiatric Association. Diagnostic and Statistical Manual of Mental Disorders. 5th ed. Washington, DC: Author; 2013.

22. Ungar M, Liebenberg L, Boothroyd R, et al. The study of youth resilience across cultures: lessons from a pilot study of measurement development. Res Hum Dev. 2008;5(3):166-188. doi:10.1080/ 15427600802274019

23. White B, Driver S, Warren AM. Resilience and indicators of adjustment during rehabilitation from a spinal cord injury. Rehabil Psychol. 2010;5(1):23-32. doi:10.1037/a0018451

24. Lei L. Empirical basis of intervention on Internet addiction among adolescents. Adv Psychol Sci. 2012;6:791-797.
25. Zhou P, Zhang C, Liu J, Wang Z. The relationship between resilience and internet addiction: a multiple mediation model through peer relationship and depression. Cyberpsychol Behav Soc Netw. 2017;20 (10):634-639. doi:10.1089/cyber.2017.0319

26. Kar SK, Arafat SY, Sharma P, Dixit A, Marthoenis M, Kabir R. COVID-19 pandemic and addiction: current problems and future concerns. Asian J Psychiatr. 2020;51:102-164. doi:10.1016/j. ajp.2020.102064

27. Contractor AA, Weiss NH, Elhai JD. Examination of the relation between PTSD symptoms, smartphone feature uses, and problematic smartphone use. Soc Sci Comput Rev. 2019;37(3):385-403. doi:10.1177/0894439318770745

28. Contractor AA, Weiss NH, Tull MT, Elhai JD. PTSD's relation with problematic smartphone use: mediating role of impulsivity. Comput Human Behav. 2017;75:177-183. doi:10.1016/j.chb.2017.05.018

29. Gros DF, Flanagan JC, Korte KJ, Mills AC, Brady KT, Back SE. Relations among social support, PTSD symptoms, and substance use in veterans. Psychol Addict Behav. 2016;30(7):764-770. doi:10.1037/ adb0000205

30. Cappell H, Greeley J. Alcohol and tension reduction: an update on research and theory. In: Blane HT, Leonard KE, editors. Psychological Theories of Drinking and Alcoholism. New York: Guilford; 1987:15-54.

31. Hébert M, Lavoie F, Blais M. Post Traumatic Stress Disorder/PTSD in adolescent victims of sexual abuse: resilience and social support as protection factors. Cien Saude Colet. 2014;19(3):685-694. doi:10.1590/1413-81232014193.15972013

32. Hsieh YP, Shen AC, Wei HS, Feng JY, Huang SC, Hwa HL. Associations between child maltreatment, PTSD, and internet addiction among Taiwanese students. Comput Human Behav. 2016;56:209-214. doi:10.1016/j.chb.2015.11.048

33. Lee JY, Kim SW, Kang HJ, et al. Relationship between problematic internet use and post-traumatic stress disorder symptoms among students following the Sewol ferry disaster in South Korea. Psychiatry Investig. 2017;14(6):871-875. doi:10.4306/ pi.2017.14.6.871

34. Schimmenti A, Passanisi A, Caretti V, et al. Traumatic experiences, alexithymia, and Internet addiction symptoms among late adolescents: a moderated mediation analysis. Addict Behav. 2017;64:314-320. doi:10.1016/j.addbeh.2015.11.002

35. Zhou X, Wu X, Zhen R. Self-esteem and hope mediate the relations between social support and post-traumatic stress disorder and growth in adolescents following the Ya'an earthquake. Anxiety Stress Coping. 2018;31(1):32-45. doi:10.1080/10615806.2017.1374376

36. Norris FH, Kaniasty K. Received and perceived social support in times of stress: a test of the social support deterioration deterrence model. J Pers Soc Psychol. 1996;71(3):498-511. doi:10.1037/00223514.71.3.498

37. Kronenberg ME, Hansel TC, Brennan AM, Osofsky HJ, Osofsky JD, Lawrason B. Children of Katrina: lessons learned about postdisaster symptoms and recovery patterns. Child Dev. 2010;81:1241-1259.

38. Wickrama KA, Kaspar V. Family context of mental health risk in tsunami-exposed adolescents: findings from a pilot study in Sri Lanka. Soc Sci Med. 2007;64(3):713-723. doi:10.1016/j. socscimed.2006.09.031

39. Brewin $\mathrm{CR}$, Andrews $\mathrm{B}$, Valentine JD, Zhou $\mathrm{X}$, Wu X, Zhen $\mathrm{R}$. Meta-analysis of risk factors for post-traumatic stress disorder in trauma-exposed adults. $J$ Consult Clin Psychol. 2000;68 (5):748-766. doi:10.1037/0022-006X.68.5.748

40. Whealin JM, Ruzek JI, Southwick S. Cognitive--behavioral theory and preparation for professionals at risk for trauma exposure. Trauma Violence Abuse. 2008;9(2):100-113. doi:10.1177/ 1524838008315869

41. Hobfoll SE. Social and psychological resources and adaptation. Rev Gen Psychol. 2002;6(4):307-324. doi:10.1037/1089-2680.6.4.307 
42. Wei DX. The Theoretical and Empirical Study of the Psychological Capital of Physical Education Teachers in Middle Schools in China [dissertation]. China: Fujian Normal University; 2012.

43. Zhu XQ. A Study of the Psychological Capital of Junior High school students and its influencing factors [dissertation]. China: Nanjing Normal University; 2010.

44. Luthans F, Avey JB, Patera JL. Experimental analysis of a web-based training intervention to develop positive psychological capital. Acad Manag Learn Educ. 2008;7(2):209-221. doi:10.5465/ amle.2008.32712618

45. Ahmad S, Feder A, Lee EJ, et al. Earthquake impact in a remote South Asian population: psychosocial factors and post-traumatic symptoms. J Trauma Stress. 2010;23(3):408-412. doi:10.1002/ jts. 20535

46. Horn SR, Charney DS, Feder A. Understanding resilience: new approaches for preventing and treating PTSD. Exp Neurol. 2016;284:119-132.

47. Ahmed AS. Post-traumatic stress disorder, resilience and vulnerability. Adv Psychiatr Treat. 2007;13(5):369-375. doi:10.1192/apt.bp.106.003236

48. Yang X, Wu X, Qi J, Zhou X. Post-traumatic stress symptoms, adversity belief, and internet addiction in adolescents who experienced a major earthquake. Curr Psychol. 2020;1-8. doi:10.1186/ s12888-021-03062-z

49. Xiao S. The theoretical basis and applications of Social Support Rating Scale (SSRS). J Clin Psychiatry. 1994;2:98-100. in Chinese.

50. Campbell SL, Stein MB. Psychometric analysis and refinement of the Connor-Davidson resilience scale (CD-RISC): validation of a 10item measure of resilience. J Trauma Stress. 2007;20(6):1019-1028. doi:10.1002/jts.20271

51. Lang AJ, Stein MB. An abbreviated PTSD checklist for use as a screening instrument in primary care. Behav Res Ther. 2005;43 (5):585-594. doi:10.1016/j.brat.2004.04.005

52. Shek DT, Tang VM, Lo CY. Internet addiction in Chinese adolescents in Hong Kong: assessment, profiles, and psychosocial correlates. Sci World J. 2008;8:776-787. doi:10.1100/tsw.2008.104

53. Liu J, Li F. Investigation of reliability and validity of the social support scale. J Xinjiang Med Univ. 2008;31(1):1-3. in Chinese.

54. Hayes AF. Introduction to mediation, moderation, and conditional process analysis: a regression-based approach. J Educ Meas. 2013;51 (3):335-337.
55. Wood EP, Cook SH. Father support is protective against the negative effects of perceived discrimination on CRP among sexual minorities but not heterosexuals. Psychoneuroendocrinology. 2019;110 (10):368-392. doi:10.1016/j.psyneuen.2019.06.019

56. Coronavirus disease (COVID-2019): situation report-72 [homepage on the Internet]. Geneva: World Health Organization; 2020. Available from: https://www.who.int/docs/default-source/coronaviruse/situa tion-reports/20200401-sitrep-72-covid-19.pdf?sfvrsn=3dd8971b_2. Accessed May 2, 2020.

57. Gunuc S, Dogan A. The relationships between Turkish adolescents' Internet addiction, their perceived social support and family activities. Comput Hum Behav. 2013;29(6):2197-2207. doi:10.1016/j. chb.2013.04.011

58. Jia Y, An L, Jia Y. The relationship between social ability and Internet Addiction of college students: the chain mediating effect of social adaptation and self-abasement. Chin J Clin Psychol. 2019;27 (1):103-107.

59. Wu Q, Luo J, Bai J, Hou M, Li X. Effect of security on mobile addiction: mediating role of actual social avoidance. Psychol Dev Educ. 2019;35(5):589-596.

60. Xu J, Ou L. Resilience and quality of life among Wenchuan earthquake survivors: the mediating role of social support. Public Health. 2014;128(5):430-437. doi:10.1016/j.puhe.2014.03.002

61. Kılınç T, Sis Çelik A. Relationship between the social support and psychological resilience levels perceived by nurses during the COVID-19 pandemic: a study from Turkey. Perspect Psychiatr Care. 2020;57(3):1000-1008. doi:10.1111/ppc.12648

62. Ye Z, Yang X, Zeng C, et al. Resilience, social support, and coping as mediators between COVID-19-related stressful experiences and acute stress disorder among college students in China. Appl Psychol Health Well Being. 2020;12(4):1074-1094. doi:10.1111/aphw.12211

63. An Y, Yuan G, Wu X, Wang W. The relationship between social support, post-traumatic stress disorder and post-traumatic growth among adolescents after wenchuan earthquake: understanding the role of self-efficacy. Psychol Dev Educ. 2018;034(1):98-104.

64. Liu S, Zhang Z. The social-psychological-physiological model of internet addiction and its research trends. Chin J Appl Psychol. 2004; 10:48-54.
Psychology Research and Behavior Management

\section{Publish your work in this journal}

Psychology Research and Behavior Management is an international, peer-reviewed, open access journal focusing on the science of psychology and its application in behavior management to develop improved outcomes in the clinical, educational, sports and business arenas. Specific topics covered in the journal include: Neuroscience, memory and decision making; Behavior modification and management; Clinical

\section{Dovepress}

applications; Business and sports performance management; Social and developmental studies; Animal studies. The manuscript management system is completely online and includes a very quick and fair peer-review system, which is all easy to use. Visit http://www. dovepress.com/testimonials.php to read real quotes from published authors. 